\title{
Atorvastatin-Induced Refractory Thrombocytopenia
}

Jasmine Ghuman ${ }^{1}$, Nicholas T. Manasewitsch ${ }^{1}$, Joban Ghuman ${ }^{2}$, Daniel Antwi-Amoabeng ${ }^{1}$, Gurpreet Chahal ${ }^{1}$

1. Internal Medicine, University of Nevada, Reno School of Medicine, Reno, USA 2. Internal Medicine, Dr. D. Y. Patil Medical College, Hospital \& Research Centre, Pimpri, IND

Corresponding author: Nicholas T. Manasewitsch, nmanasewitsch@gmail.com

\begin{abstract}
Drug-induced thrombocytopenia is rarely associated with statin medications. We describe the case of a 69year-old woman who developed refractory thrombocytopenia following atorvastatin use. To our knowledge, this is the fourth reported case of atorvastatin-induced thrombocytopenia and the first reported case of atorvastatin-induced refractory thrombocytopenia. Additionally, we summarize the cases of statin-induced thrombocytopenia reported in the medical literature.
\end{abstract}

Categories: Internal Medicine, Hematology

Keywords: thrombocytopenia, refractory, atorvastatin, drug-induced thrombocytopenia, platelet, statin-induced, hyperlipidemia, drug reaction, petechiae, purpura

\section{Introduction}

Atorvastatin is used to treat dyslipidemia and the prevention of cardiovascular and cerebrovascular diseases, particularly in people who are unable to meet their lipid-lowering goals through lifestyle modifications [13]. It inhibits 3-hydroxy-3-methyl-glutaryl-coenzyme A reductase, a key enzyme in cholesterol synthesis. The most common adverse effects of statins are dyspepsia, constipation, abdominal pain, flatulence, headache, and myalgia [4]. Myopathy, rhabdomyolysis, and liver enzyme abnormalities are rare, but major side effects seen with statin use [5,6]. A few case reports of statin-induced thrombocytopenia, specifically with atorvastatin, rosuvastatin, and simvastatin [7-15].

Review began 12/02/2020 Review ended 01/04/2021 Published 01/05/2021

\section{(c) Copyright 2021}

Ghuman et al. This is an open access article distributed under the terms of the Creative Commons Attribution License CC-BY 4.0., which permits unrestricted use, distribution, and reproduction in any medium, provided the original author and source are credited.
Thrombocytopenia can be either inherited or acquired. Acquired causes include drug-induced thrombocytopenia (DIT), viral or bacterial infections, malignancy, liver failure, and hypersplenism. Several mechanisms are proposed for DIT, but the most recent hypothesis suggests that weakly reactive platelet autoantibodies develop an increased affinity for platelet glycoprotein epitopes in the presence of the sensitizing drug [16]. Patients present with epistaxis, bruising, and petechiae [16]. In this case report, we present a probable temporal relationship between atorvastatin initiation and the onset of refractory thrombocytopenia.

\section{Case Presentation}

A 69-year-old female with a history of hyperlipidemia presented to the hospital with atraumatic bruising, traumatic right knee hematoma, and multiple painless "blood blisters" on her buccal mucosa which she noticed one day prior. Her history was negative for bleeding disorders, anticoagulant use, and antiplatelet use. She started taking atorvastatin $20 \mathrm{mg}$ daily about 10 weeks before presenting for hyperlipidemia. The patient's baseline low-density lipoprotein was between 125 and $146 \mathrm{mg} / \mathrm{dL}$ (reference range: < $100 \mathrm{mg} / \mathrm{dL}$ ) and high-density lipoprotein was between 66 and $72 \mathrm{mg} / \mathrm{dL}$ (reference range: $>40 \mathrm{mg} / \mathrm{dL}$ ). The patient was found to have profound thrombocytopenia on this admission with a platelet count of $2,000 / \mu \mathrm{L}$ (reference range: $164,000-446,000 / \mu \mathrm{L}$ ), and her other cell lines were within normal limits. Complete blood count from two months prior demonstrated a platelet count of $245,000 / \mu \mathrm{L}$. The patient was admitted for further workup.

Our patient's coagulation studies, infectious panel, and autoimmune panel (anti-nuclear antibodies, rheumatoid factor, serum immunofixation test, serum protein electrophoresis including alpha and gamma globulins) were unremarkable. She received an infusion of two units of platelets with minimal increase in her platelet count. Suspecting immune-thrombocytopenic purpura (ITP), we subsequently treated her with two doses of intravenous immunoglobulin (IVIG) over two days and oral dexamethasone 40mg for four days. Her platelet counts improved to $55,000 / \mu \mathrm{L}$, and she was discharged home with close follow-up. She continued taking atorvastatin $20 \mathrm{mg}$.

Two days after discharge, the patient returned to the hospital, presenting with worsening fatigue and purpura. She was found to have a platelet count of $1,000 / \mu \mathrm{L}$ and was readmitted. We discontinued her atorvastatin and started her on a regimen of IVIG for two days and prednisone $80 \mathrm{mg}$ for five days. Although 
she responded to this regimen, we added rituximab given our high suspicion for refractory thrombocytopenia, indicated by the lack of response to two or more treatments. Our patient's platelet count improved to $118,000 / \mu \mathrm{L}$, and she was discharged home in stable condition with instructions to complete seven days of $60 \mathrm{mg}$ of prednisone and weekly doses of rituximab. The patient was not restarted on atorvastatin or any other statin. At follow-up five months after her hospital discharge and after completing four doses of rituximab, our patient's platelet count returned to baseline, and her purpura had resolved. A timeline of the variations in our patient's platelet count is illustrated in Figure 1. She currently manages her hyperlipidemia with diet and exercise.

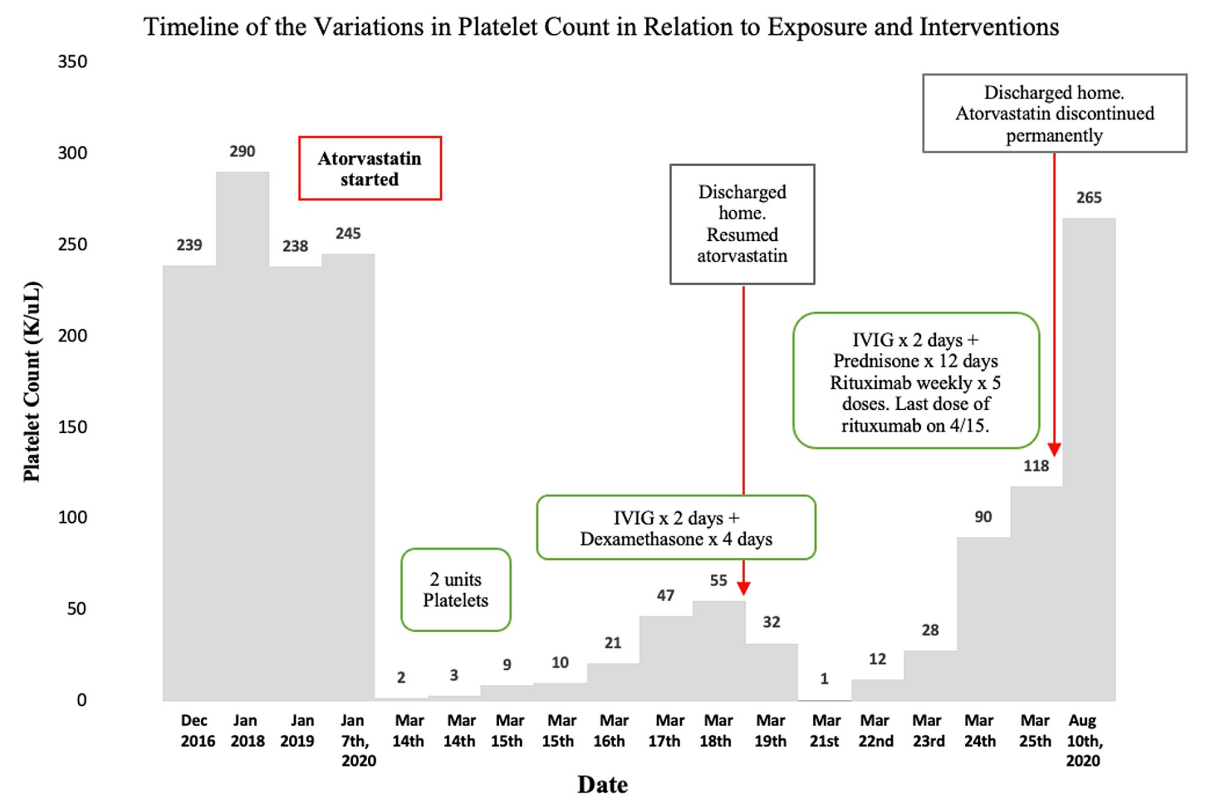

\section{FIGURE 1: Temporal variations in platelet count after initiation of atorvastatin and response to interventions.}

IVIG- Intravenous mmunoglobulin

\section{Discussion}

DIT presents with rapid symptomatic improvement following the discontinuation of the drug [16]. DIT has been associated with over 100 different mediations [16]. Two major pathologic mechanisms behind druginduced thrombocytopenia include decreased platelet production via marrow suppression and peripheral platelet clearance [10]. Patients are exposed to the implicated medication for at least a week before developing clinical signs of thrombocytopenia [16]. DIT is suggested by rapid recovery following discontinuation of the implicated medication and the temporal relationship between symptom onset and medication exposure [7]. It is common practice to discontinue the medication and treat with steroid, and IVIG and plasma exchange may be considered for refractory cases [16]. Our patient's case was considered to be refractory given that she failed two or more treatments (steroids and IVIG), and the hematology-oncology consultant on the case agreed with this determination. We recognize that ITP may present similarly and that the resolution of our patient's thrombocytopenia may be attributed to rituximab initiation, rather than atorvastatin discontinuation alone [17].

The Naranjo Algorithm is a validated standardized questionnaire of 10 question items designed to estimate the probability of a drug causing an adverse clinical event. Scores greater than 8 are considered a definite reaction, 5 to 8 considered probable, 1 to 4 considered possible, and less than 1 considered doubtful $[18,19]$. Our patient's Naranjo algorithm score of 8 (Table 1) suggests a probable association between initiation of atorvastatin and the onset of thrombocytopenia in this case. 


\section{Cureus}

Naranjo Question Item

1. Are there previous conclusive reports on this reaction?

2. Did the adverse event appear after the suspected drug was given?

3. Did the adverse reaction improve when the drug was discontinued, or a specific antagonist was given?

4. Did the adverse reaction appear when the drug was re-administered?

5. Are there alternative causes that could have caused the reaction?

6. Did the reaction reappear when a placebo was given?

7. Was the drug detected in any body fluid in toxic concentrations?

No 2

8. Was the reaction more severe when the dose was increased, or less severe when the dose was decreased?

9. Did the patient have a similar reaction to the same or similar drugs in any previous exposure?

10. Was the adverse event confirmed by any objective evidence?

TABLE 1: The Naranjo Scale for assessing the association between atorvastatin use and the adverse drug reaction (ADR) of thrombocytopenia. Our patient had a probable ADR given a score of " 8 ". The reaction followed a reasonable temporal sequence after a drug, followed a recognized response to the suspected drug, was confirmed by withdrawal but not by exposure to the drug, and could not be reasonably explained by the known characteristics of the patient's clinical state [16].

Lovastatin has been found to dose-dependently induce platelet apoptosis via mitochondrial caspase activation. In mouse models, lovastatin impairs platelet function and reduces circulating platelets in vivo, suggesting the possible pathogenesis of thrombocytopenia and hemorrhage in patients treated with statins [20]. Given these findings, it is interesting that lovastatin-induced thrombocytopenia has not yet been reported in the literature.

The reported cases of statin-induced thrombocytopenia are summarized in Table 2. It is difficult to draw conclusions from a small set of case reports; however, statin-induced thrombocytopenia has been associated with atorvastatin, rosuvastatin, and simvastatin in the literature, two of which are lipophilic molecules. Additionally, the lipophilic statin lovastatin has been demonstrated to cause platelet apoptosis in vivo. Atorvastatin-induced thrombocytopenia has been reported three times in the literature, however, our case reports a refractory presentation [9]. 


\section{Cureus}

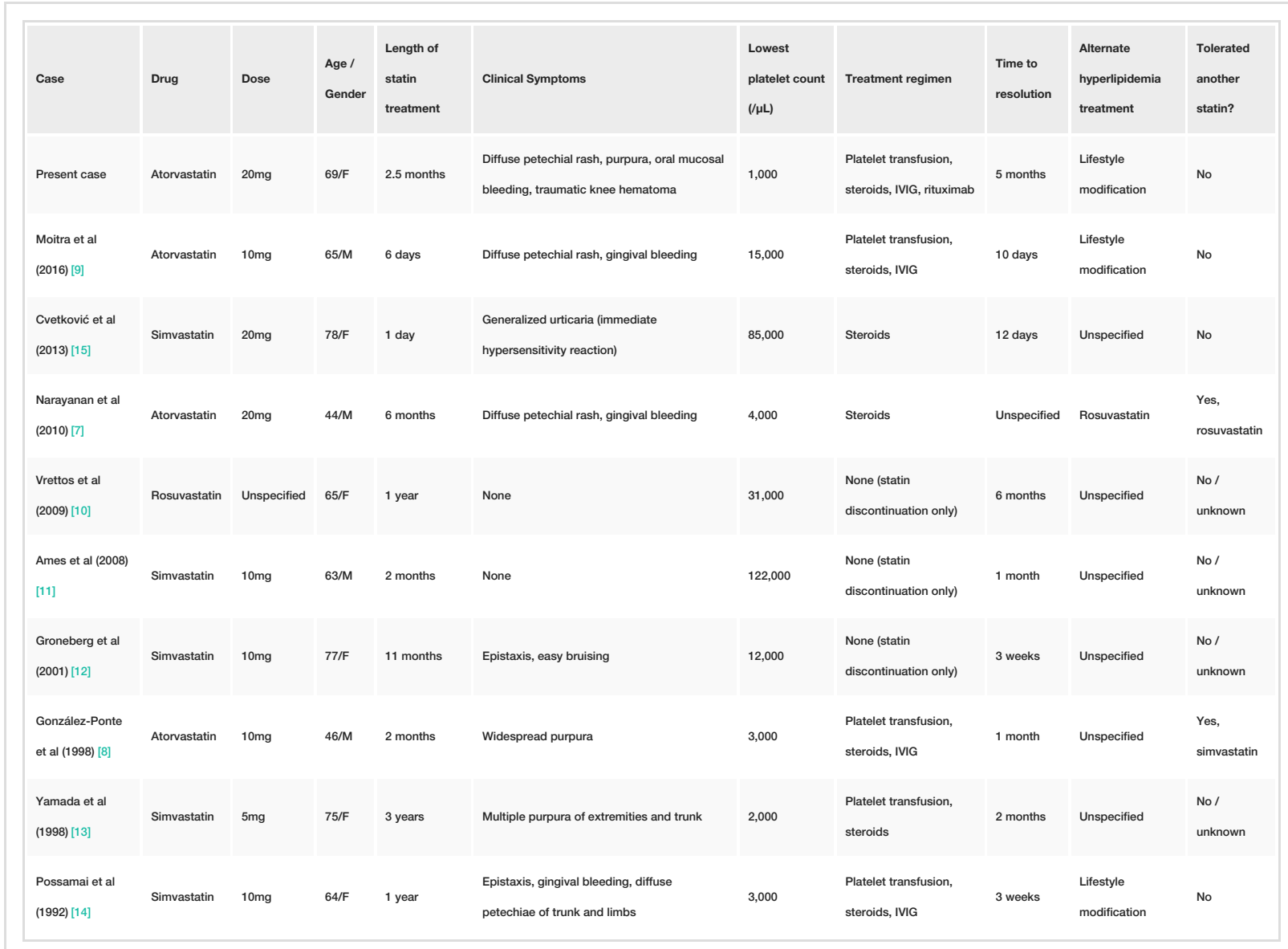

TABLE 2: Table summarizing reported cases of statin-induced thrombocytopenia in the literature.

IVIG- Intravenous immunoglobulin; M- male; F- Female

\section{Conclusions}

To our knowledge, this is the fourth reported case of atorvastatin-induced thrombocytopenia and the first reported case of atorvastatin-induced refractory thrombocytopenia. Clinicians need to be aware of this association and discontinue atorvastatin if thrombocytopenia develops. Future investigations into the relationship between statin medications and thrombocytopenia would prove useful to the medical literature.

\section{Additional Information}

\section{Disclosures}

Human subjects: Consent was obtained by all participants in this study. Conflicts of interest: In compliance with the ICMJE uniform disclosure form, all authors declare the following: Payment/services info: All authors have declared that no financial support was received from any organization for the submitted work. Financial relationships: All authors have declared that they have no financial relationships at present or within the previous three years with any organizations that might have an interest in the submitted work. Other relationships: All authors have declared that there are no other relationships or activities that could appear to have influenced the submitted work.

\section{References}

1. Diamantis E, Troupis T, Mazarakis A, Kyriakos G, Diamanti S, Troupis G, Skandalakis P: Primary and secondary prevention of acute coronary syndromes: the role of the statins. Recent Adv Cardiovasc Drug Discov. 2014, 9:97-105. 10.2174/1574890110666150708112349

2. Aznaouridis K, Masoura C, Vlachopoulous C, Tousoulis D: Statins in stroke. Curr Med Chem. 2019, 26:61746185. 10.2174/0929867326666190620104539

3. Gaspardone A, Area M: Atorvastatin: its clinical role in cerebrovascular prevention . Drugs. 2007, 67:55-62. 10.2165/00003495-200767001-00006

4. Black DM, Bakker-Arkema RB, Nawrocki JW: An overview of the clinical safety profile of atorvastatin (lipitor), a new HMG-CoA reductase inhibitor. Arch Intern Med. 1998, 158:577-584. 10.1001/archinte.158.6.577 
5. Li S, Yu Y, Jin Z, et al.: Prediction of pharmacokinetic drug-drug interactions causing atorvastatin-induced rhabdomyolysis using physiologically based pharmacokinetic modelling. Biomed Pharmacother. 2019, 119:109416. 10.1016/j.biopha.2019.109416

6. Mohamed MF, Salameh OK, Saeed AA: Statin-induced rhabdomyolysis, acute kidney injury, and hepatitis leading to death. Am J Case Rep. 2019, 20:709-712. 10.12659/AJCR.914707

7. Narayanan D, Kilpatrick ES: Atorvastatin-related thrombocytopenic purpura. BMJ Case Rep. 2010, 10.1136/bcr.01.2010.2614

8. González-Ponte ML, González-Ruiz M, Duvós E, Gutiérrez-Iñiguez MA, Olalla JI, Conde E: Atorvastatininduced severe thrombocytopenia. Lancet. 1998, 352:1284. 10.1016/S0140-6736(05)70491-8

9. Moitra S, Sen S, Das P, Banerjee I: Atorvastatin induced thrombocytopenia: a case report and review of literature. Med J DY Patil Univ. 2016, 9:117-120. 10.4103/0975-2870.167984

10. Vrettos I, Papageorgiou S, Economopoulou C, et al.: Rosuvastatin-induced thrombocytopenia. South Med J. 2010, 103:676-678. 10.1097/SMJ.0b013e3181e170f5

11. Ames PRJ: Simvastatin-induced thrombocytopaenia: a further case and a brief on its clinical relevance . Ann Hematol. 2008, 87:773-774. 10.1007/s00277-008-0480-1

12. Groneberg DA, Barkhuizen A, Jeha T: Simvastatin-induced thrombocytopenia. Am J Hematol. 2001, 67:277. 10.1002/ajh.1136

13. Yamada T, Shinohara K, Katsuki K: Severe thrombocytopenia caused by simvastatin in which thrombocyte recovery was initiated after severe bacterial infection. Clin Drug Investig. 1998, 16:172-174. 10.2165/00044011-199816020-00011

14. Possamai G, Bovo P, Santonastaso M: Thrombocytopenic purpura during therapy with simvastatin . Haematologica. 1992, 77:357-8.

15. Cvetković Z, Suvajdžić-Vuković N, Todorović Z, Panić M, Nešković A: Simvastatin and amlodipine induced thrombocytopenia in the same patient: double trouble and a literature review. J Clin Pharm Ther. 2013, 38:246-248. 10.1111/jcpt.12051

16. Aster RH, Curtis BR, McFarland JG, Bougie DW: Drug-induced immune thrombocytopenia: pathogenesis, diagnosis, and management. J Thromb Haemost. 2009, 7:911-918. 10.1111/j.1538-7836.2009.03360.x

17. Zaja F, Volpetti S, Chiozzotto M, Puglisi S, Isola M, Buttignol S, Fanin R: Long-term follow-up analysis after rituximab salvage therapy in adult patients with immune thrombocytopenia. Am J Hematol. 2012, 87:886889. 10.1002/ajh.23272

18. Naranjo CA, Busto U, Sellers EM, et al.: A method for estimating the probability of adverse drug reactions . Clin Pharmacol Ther. 1981, 30:239-245. 10.1038/clpt.1981.154

19. Murayama H, Sakuma M, Takahashi Y, Morimoto T: Improving the assessment of adverse drug reactions using the Naranjo Algorithm in daily practice: the Japan adverse drug events study. Pharmacol Res Perspect. 2018, 6:1-7. 10.1002/prp2.373

20. Zhao Q, Li M, Chen M, et al.: Lovastatin induces platelet apoptosis . Environ Toxicol Pharmacol. 2016, 42:6975. 10.1016/j.etap.2016.01.002 